\title{
ORGANISATION DES TRAVAUX
}

\section{Mandat}

Ce groupe de travail a été constitué à l'initiative de l'Insu, du Cnes et de la direction des Sciences de la matière du CEA. Le Cnes « a pour mission de développer et $d$ 'orienter les recherches scientifiques et techniques poursuivies en matière spatiale " (code de la Recherche, article L331-2 de la dernière révision de 2008), I'Insu est une tutelle pour une grande fraction des unités mixtes de recherche impliquée dans la recherche spatiale. La DSM contribue fortement à la recherche spatiale en collaboration avec le Cnes. Ces organismes ont demandé à un groupe de travail d'effectuer pour eux une réflexion sur l'avenir de la recherche spatiale en France dans le contexte de l'évolution du dispositif de recherche en cours.

L'objectif est d'anticiper les évolutions à 15 ans du dispositif scientifique national dédié à la conception, au développement, à la réalisation, au test et à l'exploitation scientifique des missions spatiales. Cela peut concerner les missions scientifiques faisant l'objet d'une programmation spécifique (ex. : le programme Cosmic Vision), l'utilisation à des fins scientifiques de données issues de missions opérationnelles, ou, dans la perspective de GMES, la réalisation de missions de démonstration associant objectifs scientifiques et anticipation de nouveaux services.

La réflexion doit porter sur l'organisation à mettre en place pour optimiser les phases critiques de réalisation technique des projets spatiaux, dont la complexité s'accroît, ainsi que les phases d'exploitation dont la durée s'allonge et le coût relatif augmente. Ces dernières nécessitent l'accès aux données, la mise en place de chaînes de traitements lourdes, et la mise à disposition d'outils d'archivage massif et de distribution. Ce schéma est amené à évolver, plus ou moins rapidement, avec les priorités instrumentales formulées par les communautés scientifiques et l'émergence de nouvelles technologies en mécanique, électronique, optique, ainsi qu'avec les nouveaux besoins logiciels. Le groupe devra également examiner la nature des métiers techniques nécessaires à chacune de ces différentes phases. Le groupe évaluera la place relative que doivent tenir, dans ces différentes phases, le Cnes, les organismes publics de recherche dotés de laboratoires directement impliqués dans les réalisations spatiales - CNRS, CEA ainsi que le rôle des organismes situés plus en aval, et celui d'autres opérateurs publics ou privés. II aura enfin à estimer l'incidence de la nouvelle organisation de gestion de la recherche, ainsi que des nouveaux outils de fédération et de coopération : RTRA, pôles de compétitivité, PRES, universités «libres et responsables »... 


\section{Objectifs}

L'exercice doit déboucher sur :

1. une cartographie nationale précise des pôles techniques nécessaires à I'accomplissement des phases amont des projets (R\&T, analyse de mission, étude de faisabilité), ainsi qu'à leur réalisation ;

2. une vision nationale des pôles thématiques nécessaires à leur exploitation et des moyens techniques (bases de données, centres informatiques...) qui doivent les accompagner ;

3. un plan pluriannuel d'emplois techniques anticipant les évolutions de métiers nécessaires au développement des technologies émergentes, tant matérielles que logicielles;

4. une réflexion sur les modèles économiques permettant de mieux cerner les coûts à achèvement des projets scientifiques, et des démonstrateurs de services, et d'optimiser ainsi les coûts totaux consolidés qui y sont dédiés.

\section{Méthodologie}

Placé sous la présidence de Jean-Loup Puget (Académie des sciences) et assisté d'un secrétaire (assuré par l'Insu : Guy Guyot), le groupe de travail a été constitué d'un noyau "institutionnel » (Cnes-Insu-CEA) et d'un ensemble d'experts scientifiques et techniques, désignés par les précédents. Les membres institutionnels du groupe de travail, appuyés sur leurs collaborateurs ont été par ailleurs chargés de fournir les documents utiles aux travaux du groupe.

\section{Calendrier des travaux}

Le groupe a été mis en place par l'Insu, le Cnes et le CEA le 15 juin 2009.

Il a tenu les réunions aux dates suivantes:

Réunion $1: 7$ juillet 2009 (Tolbiac) : revues des rapports existants sur ces sujets, discussion générales, répartition des tâches

Réunion $2: 2$ septembre 2009 (IAP) (thématique Terre, Environnement, Climat)

Réunion 3 : 25 septembre 2009 (Cnes)

Réunion $4: 28$ octobre 2009 (Sciences de l'Univers et nouvelles thématiques)

Réunion 5 : 9 novembre 2009

Réunion $6: 30$ novembre 2009

Réunion $7: 11$ janvier 2010

Réunion 8 : 2 février 2010 
Réunion 9 : 10 mars 2010

Réunion $10: 25$ mars 2010

Réunion $11: 7$ avril 2010

Réunion $12: 19$ avril 2010

Des rapports d'étape sur le travail du groupe ont été présentés au Comité des programmes scientifiques du Cnes (29 octobre 2009), au Comité interorganismes du Cnes (4 novembre 2009), au Comité scientifique de I'Insu (17 décembre 2009), au Conseil de l'Insu (18 décembre 2009) et aux directeurs de laboratoires en sciences de l'Univers et physique fondamentale impliqués dans la recherche spatiale. Les avis et commentaires reçus ont été pris en compte.

Le groupe a consulté des experts à la CPU et l'Onera.

Des délégations du groupe ont entendu quelques industriels fortement impliqués dans les projets scientifiques spatiaux, soit sur la réalisation des charges utiles soit sur l'exploitation des données.

À l'Académie des sciences, le rapport a été soumis au Groupe de lecture critique le 27 mai 2010 et adopté par l'assemblée plénière le 29 juin 2010. 
This page intentionally left blank 